\title{
CENTRO CIRÚRGICO: RECOMENDAÇÕES PARA 0 ATENDIMENTO DE PACIENTES COM SUSPEITA OU PORTADORES DE COVID-19
}

Surgical Center: recommendations for the care of patients with suspected or confirmed COVID-19

Centro quirúrgico: recomendaciones para el cuidado de pacientes con COVID-19 sospechado o confirmado

Denilse Damasceno Trevilato ${ }^{1 *}$ (D), Marielli Trevisan Jost ${ }^{2}$ (D), Bárbara Rodrigues Araujo ${ }^{3}$ (D), Fabiana Zerbieri Martins ${ }^{4}$ (D), Ana Maria Müller de Magalhães ${ }^{5}$ (D) Rita Catalina Aquino Caregnato ${ }^{6}$ if

RESUMO: Objetivo: Apresentar as recomendações para reorganização do centro cirúrgico no atendimento a pacientes com suspeita ou confirmação de COVID-19. Método: Revisão crítica da literatura, com publicações dos anos de 2019 e 2020 relacionadas à produção científica, a normas técnicas, às diretrizes e recomendações de sociedades, para o gerenciamento do centro cirúrgico na assistência a pacientes durante a pandemia do novo coronavírus. Resultados: A gestão dos recursos humanos e materiais é primordial para: atender à demanda assistencial perioperatória; reorganizar os procedimentos cirúrgicos; garantir a segurança dos profissionais de saúde; organizar a sala cirúrgica com materiais necessários; planejar a recuperação pós-anestésica do paciente; e realizar limpeza e desinfecção da sala cirúrgica. Conclusão: As recomendações apresentadas direcionam os enfermeiros a aplicar as melhores práticas no atendimento aos pacientes, em consonância com evidências científicas recomendadas por instituições de referência, para promoção de assistência segura e de qualidade aos pacientes e profissionais. Palavras-chave: Centros cirúrgicos. Assistência perioperatória. Gestão de recursos da equipe de assistência à saúde. Pandemias. Infecções por coronavírus.

ABSTRACT: Objective: To present the recommendations to reorganize surgical center in the care of patients with suspected or confirmed COVID-19. Method: Critical literature review of the literature, with publications from 2019 and 2020 related to scientific production, technical standards, guidelines and recommendations of societies for managing the surgical center in the care of patients during the new coronavirus pandemic. Results: The management of human and material resources is essential to meet the perioperative care demand; reorganize surgical procedures; ensure the safety of health professionals; organize an operating room with required materials; plan the patient's post-anesthetic recovery; and perform cleaning and disinfection of the operating room. Conclusion: The recommendations direct nurses to apply best practices in patient care, in line with scientific evidence recommended by reference institutions, to promote safe and quality care for patients and professionals. Keywords: Surgicalcenters. Perioperative care. Crew resource management, healthcare. Pandemics. Coronavirus infections.

RESUMEN: Objetivo: presentar las recomendaciones para la reorganización del Centro Quirúrgico en la atención de pacientes con COVID-19 sospechado o confirmado. Método: Revisión crítica de la literatura, con publicaciones de los años 2019 y 2020, relacionadas con producción científica, estándares técnicos, Guías y Recomendaciones de Sociedades, para el manejo del CQ en la asistencia a pacientes durante la pandemia del nuevo coronavirus. Resultados: La gestión de los recursos humanos y materiales es fundamental para atender la demanda de cuidados perioperatorios, reorganizar los procedimientos quirúrgicos; garantizar la seguridad de los profesionales de la salud; organizar el quirófano con los materiales necesarios; planificar la recuperación postanestésica del paciente y realizar la limpieza y desinfección del quirófano. Conclusión: Las recomendaciones presentadas a enfermeras directas para aplicar las mejores prácticas en la atención al paciente, en línea con la evidencia científica recomendada por las instituciones de referencia, para promover una atención segura y de calidad a pacientes y profesionales. Palabras clave: Centros quirúrgicos. Atención perioperativa. Gestión de recursos de personal en salud. Pandemias. Infecciones por coronavirus.

'Doutoranda em Enfermagem pelo Programa de Pós-Graduação em Enfermagem (PPGENF) da Universidade Federal do Rio Grande do Sul (UFRGS) - Porto Alegre (RS), Brasil. ${ }^{2}$ Enfermeira assistencial da Santa Casa de Misericórdia de Porto Alegre - Porto Alegre (RS), Brasil.

${ }^{3}$ Acadêmica do curso de Bacharelado em Enfermagem, Universidade Federal de Ciências da Saúde de Porto Alegre (UFCSPA) - Porto Alegre (RS), Brasil.

${ }^{4}$ Enfermeira Assistencial II na Unidade de Recuperação Pós-Anestésica do Hospital de Clínicas de Porto Alegre - Porto Alegre (RS), Brasil.

${ }^{5}$ Professora associada do Departamento de Assistência e Orientação Profissional da Escola de Enfermagem e professora permanente do PPGENF da UFRGS - Porto Alegre (RS), Brasil.

'Professora adjunta IV do Departamento de Enfermagem da UFCSPA e docente do curso de Bacharelado em Enfermagem da UFCSPA - Porto Alegre (RS), Brasil.

*Autora correspondente: denilset@hotmail.com

Recebido: 01/06/2020 - Aprovado: 22/08/2020

https://doi.org/10.5327/Z1414-4425202000030009 


\section{INTRODUÇÃO}

Dezembro de 2019 ficará marcado na história da humanidade como o mês em que se desencadeou, na cidade de Wuhan, na China, uma série de casos de pneumonia de etiologia desconhecida que culminaria em uma pandemia com características ainda não vivenciadas no século XXI. Com o sequenciamento do genoma do agente viral pelo Centro Chinês para Controle e Prevenção de Doenças, foi possível identificar o vírus como pertencente à família Coronavírus, posteriormente nomeado como severe acute respiratory syndrome coronavirus 2 (SARS-CoV-2) ${ }^{1}$. No período entre dezembro de 2019 e março de 2020, a coronavirus disease (COVID-19) atingiu todos os continentes, tornando-se uma pandemia ${ }^{2}$, com os primeiros casos registrados no Brasil no mês de fevereiro do ano de $2020^{3}$.

A Organização Mundial da Saúde (OMS) denominou a doença de COVID-19 e classificou-a em duas formas diferentes ou complementares de manifestação $0^{4-6}$. Essa doença pode se manifestar por sintomas mais brandos, como febre, tosse, dor de garganta e cefaleia; ou por síndrome respiratória aguda grave (SRAG), apresentando sintomas mais severos, como dispneia, hipoxemia e taquipneia, conduzindo para o desfecho morte ${ }^{7}$. Portanto, a sintomatologia pode evoluir, levando os pacientes à internação hospitalar e ao tratamento em unidades de terapia intensiva (UTI) ${ }^{1}$.

Diante do contexto de uma crise global ocasionada pela pandemia, a reorganização dos serviços de saúde para atender à crescente demanda assistencial tornou-se iminente $e^{8,9}$. Em nível mundial, as instituições de saúde estão se mobilizando e construindo protocolos de segurança para o atendimento aos pacientes com suspeita ou portadores de COVID-19, considerando como principal via de transmissão o contato das vias aéreas, por gotículas ou aerossóis ${ }^{4,6,10}$. Os procedimentos de manipulação do trato respiratório, como intubação, aspiração endotraqueal e extubação, envolvendo aerossolização de partículas, são avaliados como de alto risco e podem infectar os profissionais da saúde ${ }^{5,6,11}$.

O afastamento e a morte de vários profissionais da saúde, em todo o mundo, principalmente daqueles que estão na linha de frente, como profissionais de enfermagem e médicos, mobilizaram conselhos federais no Brasil, que elaboraram e publicaram recomendações específicas para evitar a contaminação desses profissionais. O Conselho Federal de Enfermagem (Cofen) publicou duas versões das recomendações gerais para organização dos serviços de saúde e preparo das equipes de enfermagem durante a pandemia ${ }^{12}$. No que tange aos procedimentos anestésico-cirúrgicos, as escalas de agendamento têm a orientação de atender a intervenções com caráter de urgência e emergência, para a priorização do atendimento às vítimas da COVID-19 $9^{9,13,14}$.

Associações internacionais da área cirúrgica, tais como American College of Surgeons, American Society of Anesthesiologists, Association of periOperative Registered Nurses e American Hospital Association, também recomendaram a suspensão das cirurgias eletivas no período de pandemia da COVID-19 e se uniram, elaborando uma declaração conjunta que contém um roteiro com princípios e questões a serem avaliados no planejamento da retomada de cirurgias eletivas $^{15}$. Esse roteiro recomenda o retorno das cirurgias eletivas somente se a cidade atender aos seguintes critérios:

- redução sustentada de novos casos de COVID-19 na área geográfica por pelo menos 14 dias;

- autorização pelas autoridades sanitárias;

- capacidade de tratar com segurança todos os pacientes que necessitem de internação;

- números adequados de leitos;

- disponibilização de equipamentos de proteção individual (EPI) suficientes para a equipe de trabalho;

- previsão e abastecimento de medicações e suprimentos necessários;

- número adequado de funcionários capacitados para os atendimentos, sem comprometimento da segurança ${ }^{15}$.

Considera-se o enfermeiro como o profissional fundamental para a reorganização da assistência perioperatória, atendendo à demanda excepcional e necessária para oferecer segurança aos profissionais e aos pacientes durante essa emergência de saúde pública. Dessa forma, entende-se que é importante fazer uma síntese das publicações recentes com orientações das práticas essenciais no centro cirúrgico (CC), para auxiliar os enfermeiros nessa força-tarefa. Para isso, buscou-se revisar as recomendações indispensáveis à adequação do CC no atendimento a pacientes com suspeita ou confirmação de COVID-19.

Diante desse contexto, emergiu como pergunta de pesquisa: quais são as melhores práticas referidas na literatura sobre o atendimento a portadores de COVID-19 no CC?

\section{OBJETIVO}

Apresentar as recomendações existentes para a reorganização do CC no atendimento a pacientes com suspeita ou 
confirmação de COVID-19, garantindo segurança aos pacientes e à equipe multiprofissional.

\section{MÉTODO}

Trata-se de uma revisão crítica da literatura, com publicações dos anos de 2019 e 2020 relacionadas à produção científica, a normas técnicas, bem como às diretrizes e recomendações de sociedades, para o gerenciamento do CC na assistência a pacientes durante a pandemia do novo coronavírus. O ponto de vista crítico centralizou-se na análise das recomendações emanadas nessa produção, pretendendo conhecê-las para fundamentar a reorganização do CC no atendimento a pacientes com suspeita ou confirmação de COVID-19.

\section{RESULTADOS}

Foram selecionadas recomendações publicadas pelos seguintes órgãos, sociedades ou associações, nacionais e internacionais:

- Associação Brasileira de Enfermeiros de Centro Cirúrgico, Recuperação Anestésica e Centro de Material e Esterilização;

- Association of periOperative Registered Nurses (AORN);

- Agência Nacional de Vigilância Sanitária (ANVISA);

- Sociedade Brasileira de Anestesiologia;

- American College of Surgeons;

- American Society of Anesthesiologists;

- American Hospital Association;

- Centers for Disease Control and Prevention.

Visto que a construção deste artigo deu-se no início da pandemia, quando, portanto, a temática era nova, selecionaram-se publicações recentes de periódicos científicos reconhecidos internacionalmente, totalizando cinco artigos, que fundamentaram a análise crítica.

Após leitura dos materiais selecionados, identificaram-se as recomendações existentes para reorganização do CC no atendimento aos pacientes com suspeita ou confirmação de COVID-19, resultando nas seguintes temáticas abordadas:

- reorganização dos procedimentos cirúrgicos;

- segurança dos profissionais de saúde;

- organização de sala cirúrgica;

- recuperação pós-anestésica do paciente;

- limpeza e desinfecção da sala cirúrgica.

\section{DISCUSSÃO}

\section{Reorganização dos procedimentos cirúrgicos: uma nova realidade}

Para organização dos CC de todo o Brasil durante a pandemia da COVID-19, uma série de discussões sobre as recomendações da comunidade científica mundial aponta para a suspensão de procedimentos eletivos, diminuindo o contingente de circulação nas instituições e priorizando pacientes de procedimentos de urgência, emergência e oncológicos ${ }^{13-15}$.

Outras ações relevantes são disponibilizar os insumos em saúde necessários - tais como máscaras cirúrgicas, máscaras N95, aventais de proteção individual e materiais de assistência ventilatória ${ }^{8}$ - e otimizá-los para o atendimento de pacientes portadores de COVID-19, tanto no processo de triagem destes quanto na proteção dos profissionais envolvidos.

Considerando que a COVID-19 é uma doença nova, ela requer ainda o monitoramento epidemiológico para se conhecer seu potencial de contaminação para além do trato respiratório. Portanto, ainda demanda pesquisas que identifiquem fatores etiológicos relacionados ao seu desenvolvimento e à sua prevenção ${ }^{8}$.

Há sugestão de que pacientes com COVID-19 positivo não realizem cirurgias nesse momento, a menos que tenham uma emergência com risco de morte, ou caso a cirurgia não possa ser adiada. Nos casos de pacientes com status de COVID-19 desconhecido, o ideal é fazer o teste pré-operatório e, no caso de atendimento, sempre utilizar $\mathrm{EPI}^{8,13}$. A difícil decisão de adiar o procedimento muitas vezes é essencial diante dessa situação global ${ }^{9}$ e traz benefícios tanto aos profissionais de saúde quanto aos pacientes ${ }^{16}$.

Nas cirurgias laparoscópicas, a manutenção do pneumoperitônio artificial pode expor a equipe cirúrgica ao risco de contaminação com aerossóis. Estudos anteriores referentes a outros vírus comprovam que há dispersão desses organismos por meio dos gases de expulsão do pneumoperitônio e pelos trocateres ${ }^{8,16,17}$. Manejo especial deve ser adotado durante a laparoscopia, com controle da pressão do pneumoperitônio ao mínimo possível, sem que, no entanto, haja comprometimento da visão do campo cirúrgico. Da mesma forma, devem-se utilizar o cautério em menor potência e a aspiração com filtragem durante a remoção da fumaça e aerossóis ${ }^{8,14,16-18}$. 


\section{Segurança dos profissionais de saúde}

$\mathrm{Na}$ organização das instituições de saúde para atender aos procedimentos anestésico-cirúrgicos de pacientes suspeitos e confirmados de COVID-19, deve-se considerar, na equipe de saúde, descartar a participação de funcionários dos grupos de risco (diabetes, hipertensão e doenças cardiovasculares $^{6,12}$, idade superior a 60 anos, doenças respiratória ou renal crônicas, portadores de tuberculose e hanseníase ou outras doenças infecciosas crônicas, transplantados de órgãos sólidos e de medula óssea, imunossupressão por doenças e / ou medicamentos, portadores de doenças cromossômicas e com estados de fragilidade imunológica, além das gestantes $)^{12}$. Recomenda-se revezamento entre os funcionários para atendimento a esses pacientes, bem como realocação de colaboradoras grávidas e lactantes para que não haja risco de contato direto com pacientes suspeitos ou confirmados para infecção por coronavírus ${ }^{12}$.

Para proteção em relação ao risco de contaminação, deve ser vetado o uso de qualquer tipo de adorno (alianças, correntes, anéis, relógios, brincos e crachás pendurados em cordão), por dificultarem a higienização adequada das mãos e propiciarem o acúmulo de microrganismos ${ }^{6,12}$.

Os profissionais devem ter adequada capacitação e compreensão para o uso e a retirada de $\mathrm{EPIs}^{6,13}$, touca, avental descartável ou capote, máscara N95, óculos ou proteção facial, luvas e sapatos fechados e impermeáveis ${ }^{6,11,12,16,17}$. Apesar de a ANVISA não recomendar o uso de duas luvas (duplo enluvamento) $)^{5}$, uma revisão sistemática concluiu que não há evidências de que a proteção adicional reduza a infecção no paciente, entretanto o segundo par de luvas diminui significativamente as perfurações nas luvas internas ${ }^{19}$, podendo se tornar uma proteção à equipe cirúrgica. Recomenda-se a utilização de duas luvas como forma de reduzir o risco de autocontaminação, realizando a troca do par de luvas externas sempre que houver contato com sangue e fluidos, mantendo o par de luvas internas, se estiverem limpas ${ }^{11}$. A AORN orienta que todos os membros paramentados da equipe cirúrgica utilizem dois pares de luvas estéreis ${ }^{20}$. Por sua vez, as autoras deste artigo acreditam na seguinte máxima: é melhor pecar por excesso do que por falta. A higienização das mãos com água e sabão ou álcool 70\% deve ser executada antes e depois da colocação dos EPI, nos cinco momentos preconizados pela OMS: antes de tocar no paciente, antes de realizar procedimentos assépticos, após contato com o paciente, após o risco de exposição a fluidos corporais e após contato com áreas próximas ao paciente $e^{5,6}$.
As máscaras N95 devem ser utilizadas, pois oferecem proteção em relação aos aerossóis ${ }^{6,10,13,15,16}$. Seu uso prolongado, ou seja, a não remoção do dispositivo por seis a oito horas, pode ser implantado quando em atendimento de pacientes com COVID-19 $9^{6,21}$. No que diz respeito ao polêmico aspecto do reúso dessas máscaras, ainda não existe consenso quanto ao número de vezes seguro para a reutilização. Algumas considerações desencorajam o reúso, pelo risco de contaminação do profissional na manipulação do dispositivo ${ }^{6}$. Entretanto, diante do contexto de racionamento e otimização de insumos de saúde, os profissionais necessitam de capacitação para a execução adequada do reúso de tais máscaras, conforme recomendações vigentes ${ }^{21}$. A máscara deve ser trocada após a realização de procedimentos geradores de aerossóis, quando houver contaminação por sangue e outras secreções, ou depois de atendimento de paciente com coinfecção que demande precaução de contato ${ }^{6}$.

Ante o agendamento de procedimentos e a organização da escala de trabalho, desde a admissão no CC, os profissionais precisam estar utilizando todos os EPIs para receber o paciente portador de COVID-19, que deverá ser levado diretamente para a sala cirúrgica, sem permanência em recepções ou salas de pré-operatório ${ }^{6}$. Durante o procedimento, todos os profissionais devem estar com os EPIs completos, manter as portas da sala cirúrgica fechadas ao longo do procedimento e colocar na porta da sala uma identificação alertando para protocolo de paciente com COVID- $19^{6}$.

Observa-se a importância de ter atenção especial na retirada dos EPIs, pelo risco de contaminação do profissional ${ }^{6}$. Conforme as recomendações da ANVISA ${ }^{5}$, a desparamentação deve ser feita da seguinte maneira:

- ainda dentro da sala: retirar luvas, avental, higienizar as mãos;

- ao sair da sala: higienizar as mãos, retirar gorro, óculos de proteção ou protetor facial, higienizar as mãos, retirar a máscara N95, higienizar as mãos;

- ao final: higienizar os óculos de proteção ou protetor facial.

Após o procedimento, todos os profissionais devem tomar banho antes de continuar suas funções ${ }^{6,16}$.

Para transportar o paciente, os profissionais precisam estar usando EPI e o paciente deve utilizar máscara cirúrgica $^{5}$. Nos procedimentos com aerossolização de secreções respiratórias, é impreterível o uso de máscara N95 ${ }^{5,11}$. 


\section{Organização de sala cirúrgica}

No momento do agendamento cirúrgico, deve-se informar a respeito de pacientes suspeitos ou confirmados para infecção pelo coronavírus ${ }^{6,13}$. Sugere-se utilizar a mesma sala cirúrgica para atendimento a pacientes com suspeita ou confirmação de COVID-19 até o fim da pandemia e garantir que se tenha intervalo mínimo de agendamento de uma hora entre dois procedimentos, tempo este destinado aos cuidados com higienização, ${ }^{6,16}$.

Recomenda-se disponibilizar a mesma sala cirúrgica e o mesmo aparelho de anestesia específico para atendimento a esses pacientes até o fim da pandemia ${ }^{6,11}$. Procedimentos com geração de aerossóis, como intubação, devem ser realizados, preferencialmente, em sala com pressão negativa ou ar-condicionado desligado (pressão neutra) $)^{5,6,10,17}$.

A intubação deve ser feita pelo profissional mais experiente, por indução com sequência rápida, para evitar a ventilação manual sob máscara do paciente. Deve-se evitar a intubação com o paciente acordado e devem ser utilizados circuitos de aspiração fechados ${ }^{11}$. Recomenda-se o emprego de filtro bacteriano / viral com eficiência superior a 99,5\% HMEF (trocador de calor e umidade) de barreira, conectado entre o tubo do paciente e o circuito de ventilação. Se possível, usar filtro bacteriano para filtragem do ar expirado, para caso de falhas do filtro próximo ao paciente, e um terceiro filtro bacteriano na linha do ar inspirado $^{6,10}$. Se disponível, deverá ser utilizado videolaringoscópio, a fim de minimizar a proximidade com a face do paciente ${ }^{11,13}$. Em necessidade de troca do ventilador, faz-se preciso uma pinça de preensão para ocluir o tubo e evitar a geração de aerossóis ${ }^{6,11,13}$.

Priorizar equipamentos e materiais descartáveis em sala cirúrgica, com montagem de kits. Todo material não utilizado que entrou em sala cirúrgica deverá ser descartado ${ }^{6}$. Manter em sala cirúrgica somente o estritamente necessário. Materiais que permanecerem na sala cirúrgica, como equipamentos, aparelho de anestesia e monitores, devem ser cobertos com campos impermeáveis e descartáveis, a fim de reduzir a contaminação e facilitar a limpeza ${ }^{6,22}$.

Toda a equipe deve usar os EPIs de maneira adequada para recepção do paciente, encaminhado diretamente para a sala cirúrgica, utilizando a comunicação efetiva entre todos os seus membros ${ }^{6,11}$. Quanto ao número de profissionais, recomenda-se restringi-lo ao estritamente necessário ao procedimento executado ${ }^{6,14}$, indicando-se disponibilizar um técnico de enfermagem circulante na área externa para atender à sala cirúrgica e promover melhor adesão às recomendações, bem como dispor, dentro da sala cirúrgica, somente o material de fato essencial ${ }^{5,6,13,16}$.

\section{Recuperação pós-anestésica do paciente}

Diante do risco de disseminação da COVID-19, os pacientes com diagnóstico suspeito ou confirmado do vírus que não necessitem ser transferidos para UTI para recuperação devem permanecer na própria sala operatória, até sua completa recuperação, visto que a equipe já está paramentada adequadamente com os EPIs para a assistência a ser realizada, a fim de evitar o contato com outras pessoas ${ }^{6,11,16}$.

Recomendações orientam que o paciente se mantenha durante toda a sua recuperação e seja transportado diretamente para a unidade de destino com máscara cirúrgica e, se necessário, com suporte de oxigênio, que deve ficar embaixo da máscara ${ }^{6}$. Após a alta do CC, os aventais e as luvas utilizados pelos profissionais devem ser retirados dentro da sala cirúrgica ${ }^{6,16}$.

\section{Limpeza e desinfecção da sala cirúrgica}

A limpeza da sala deve ser iniciada somente após a saída do paciente ${ }^{18}$. Na desmontagem, é recomendado o duplo enluvamento, devendo a primeira luva ser trocada sempre que houver contato com material contaminado ${ }^{6}$. Cabe à equipe de enfermagem organizar instrumentais em recipientes plásticos hermeticamente fechados e identificados, para prevenir possíveis contaminações, e destiná-los ao centro de materiais e esterilização da instituição ${ }^{5,6}$, bem como realizar troca de todo o circuito de via aérea, cal sodada, filtros e desinfecção do aparelho de anestesia e do cânister da cal sodada ${ }^{6,11}$.

Para a limpeza da sala cirúrgica, indica-se a utilização completa dos EPIs para precaução de contato e aerossóis, mantendo pressão negativa ou ar-condicionado desligado ${ }^{6}$. Deve-se realizar limpeza minuciosa de superfícies e equipamentos ${ }^{5,13}$. A desinfecção das superfícies só deve ser executada após a sua limpeza $^{5}$. A limpeza de superfícies deve ocorrer com detergente neutro, seguida de desinfecção ${ }^{13}$. A desinfecção pode ser feita com álcool 70\%, hipoclorito de sódio, aqueles à base de quaternário de amônia, ou outro desinfetante indicado para esse fim $^{5,12,13,23}$. Há recomendações específicas para limpeza e desinfeç̧ão de superfícies que tiveram contato com paciente em suspeita ou confirmação de COVID-19 com soluções à base de quaternário de amônia ou hipoclorito de sódio ${ }^{6}$.

Orienta-se realizar a limpeza com o máximo de eficiência, com atenção especial às superfícies próximas ao paciente (mesa cirúrgica, cadeiras, entre outras), incluindo equipamentos eletrônicos (bombas de infusão, monitores, telas, cabos e outros), mobiliário ${ }^{5,6,16,22}$, aparelho de anestesia ${ }^{6,22}$, sobretudo em áreas frequentemente tocadas (interruptores, maçanetas, botões e 
controles) ${ }^{22}$. As sujidades visíveis (sangue e secreções) devem ser removidas com papel toalha e limpas antes da desinfecção ${ }^{16}$.

Estabelecer método e sequência de limpeza unidirecional, do local mais limpo para o mais sujo ${ }^{22}$. Após a limpeza de equipamentos e superfícies, deve ser realizada limpeza terminal pela equipe de higienização, incluindo paredes e chão ${ }^{18}$. Uma lista de verificação, tipo checklist, deve ser utilizada como forma de padronizar e garantir que todos os itens e procedimentos de limpeza foram executados ${ }^{22}$.

Não existem recomendações especiais quanto à lavagem de roupas contaminadas, mas deve-se ter cuidado no transporte e no manuseio delas ${ }^{5,22}$.

Todos os resíduos dos procedimentos assistenciais devem ser considerados na categoria A1, conforme Resolução da Diretoria Colegiada (RDC) $n^{\circ} 222$, de 28 de março de 2018, da ANVISA $A^{6,22,24}$, e descartados em saco específico como resíduo infectante ${ }^{5,6}$.

\section{Limitações do estudo}

Com a excepcionalidade do cenário atual, identifica-se como limitação do estudo a atualização constante de publicações relacionadas ao tema, estas restritas a recomendações de sociedades e especialistas, bem como fundamentadas em estudos com base em epidemias passadas. Contudo, ainda são escassas pesquisas sobre o novo coronavírus e a efetividade das medidas recomendadas pelos especialistas no panorama da pandemia da COVID-19.

\section{Contribuições para a prática}

Tendo em vista o cenário pandêmico atual, que demanda dos serviços de saúde a reorganização de seus fluxos de trabalho, faz-se necessário que os profissionais tenham conhecimento suficiente sobre as medidas de prevenção e controle. Dessa forma, este estudo vem contribuir como uma síntese da literatura, trazendo informações e evidências fundamentais, bem como subsídios, para a manutenção da saúde e a segurança durante a pandemia de COVID-19 no âmbito perioperatório.

\section{CONSIDERAÇÕES FINAIS}

Com a revisão das recomendações das associações de referência e de evidências científicas, identificou-se a necessidade de reorganização do serviço no CC para a assistência a pacientes com suspeita ou confirmação da doença durante a pandemia de COVID-19, garantindo segurança aos pacientes e à equipe multiprofissional.

Entre as recomendações indicadas, destaca-se a gestão dos recursos humanos e materiais para atender à demanda assistencial perioperatória, com reorganização dos procedimentos cirúrgicos eletivos, garantia da segurança dos profissionais de saúde, organização da sala cirúrgica com materiais necessários, planejamento da recuperação pós-anestésica do paciente e realização da limpeza e desinfecção da sala cirúrgica.

Pretende-se que este artigo possa contribuir, de forma a compartilhar com os profissionais da área, com as principais recomendações atualizadas para a assistência no contexto perioperatório. Dessa maneira, busca-se atendimento de qualidade e seguro para todos os indivíduos envolvidos nesse processo, desde o agendamento da cirurgia até a recuperação pós-cirúrgica.

\section{REFERÊNCIAS}

1. Huang C, Wang Y, LiX, Ren L, Zhao J, Hu Y, et al. Clinical features of patients infected with 2019 novel coronavirus in Wuhan, China. The Lancet [Internet]. 2020 [accessed on Apr 1st, 2020];395:497-506. Available at: https://www. thelancet.com/action/showPdf?pii=S0140-6736\%2820\%2930183-5. https://doi.org/10.1016/S0140-6736(20)30183-5

2. Kraemer MUG, Yang CH, Gutierrez B, Wu C, Klein B, Pigott DM, et al. The effect of human mobility and control measures on the COVID-19 epidemic in China. Science [Internet]. 2020 [accessed on Apr 1st, 2020];368(6490):4937. Available at: https://science.sciencemag.org/content/early/2020/03/25/ science.abb4218. https://doi.org/10.1126/science.abb4218
3. Rodriguez-MoralesAJ,GallegoV,Escalera-Antezana JP, MéndezCA,Zambrano LI, Franco-Peredes C, et al. COVID-19 in Latin America: The implications of the first confirmed case in Brazil. Travel Med Infect Dis [Internet]. 2020 [accessed on Apr 1st, 2020];35:101613.Available at:https://www.ncbi.nlm.nih.gov/pmc/ articles/PMC7129040/. https://dx.doi.org/10.1016\%2Fj.tmaid.2020.101613

4. World Health Organization (WHO). Director-General's remarks at the media briefing on 2019-nCoV [Internet]. World Health Organization; 2020 [accessed on Apr 3rd, 2020]. Available at: https://www.who. $\mathrm{int} / \mathrm{dg} /$ speeches/detail/who-director-general-s-remarks-at-themedia-briefing-on-2019-ncov-on-11-february-2020 
5. Agência Nacional de Vigilância Sanitária (ANVISA). Nota técnica GVIMS/ GGTES/ANVISA n० 04/2020 - Orientações para os serviços de saúde: medidas de prevenção e controle que devem ser adotadas durante a assistência aos casos suspeitos ou confirmados de infecção pelo novo coronavírus (SARS-CoV-2) [Internet]. Brasília: ANVISA; 2020 [accessed on Apr 3rd, 2020]. 53 p. Available at: https://www20.anvisa. gov.br/segurancadopaciente/index.php/alertas/item/nota-tecnican-04-2020-gvims-ggtes-anvisa-atualizada-em-21-03-2020

6. Associação Brasileira de Enfermeiros de Centro Cirúrgico, Recuperação Anestésica e Centro de Material e Esterilização (SOBECC). Recomendações relacionadas ao fluxo de atendimento de pacientes com suspeita ou infecção confirmada pelo COVID-19 em procedimentos cirúrgicos ou endoscópicos [Internet]. $2^{\mathrm{a}}$ ed. São Paulo: SOBECC; 2020 [accessed on Apr 3rd, 2020]. 14 p. Available at: http://sobecc.org.br/ arquivos/RECOMENDACOES_SOBECC_COVID_20_EDICAO_.pdf

7. Brasil. Ministério da Saúde. Secretaria de Atenção Especializada à Saúde. Departamento de Atenção Hospitalar, Domiciliar e de Urgência. Protocolo de manejo clínico da Covid-19 na Atenção Especializada [Internet]. Brasília: Ministério da Saúde; 2020 [accessed on Apr 7, 2020]. 48 p. Available at: https://portalarquivos.saude.gov.br/images/ pdf/2020/April/14/Protocolo-de-Manejo-Cl--nico-para-o-Covid-19.pdf

8. Cohen SL, Liu G, Abrao M, Smart N, Heniford T. Perspectives on Surgery in the time of COVID-19: Safety First. J Minim Invasive Gyneco. [Internet]. 2020 [accessed on Apr 10, 2020];27(4):792-3. Available at: https://doi.org/10.1016/j.jmig.2020.04.003

9. Woodson E, Sydlowski S. CI Surgery Cancellations due to COVID-19. Hear J [Internet]. 2020 [accessed on Apr 15, 2020];73(4):38-9. Available at: https://journals.lww.com/thehearingjournal/fulltext/2020/04000/ ci_surgery_cancellations_due_to_covid_19.14.aspx. https://doi. org/10.1097/01.HJ.0000661624.87101.5a

10. Infectious Diseases Society of America (IDSA). Infectious Diseases Society of America Guidelines on Infection Prevention for Health Care Personnel Caring for Patients with Suspected or Known COVID-19 [Internet]. Arlington: IDSA; 2020 [accessed on Apr 28, 2020]. Available at: www.idsociety.org/COVID19guidelines/ip

11. Sociedade Brasileira de Anestesiologia (SBA). 0 coronavírus e o anestesiologista [Internet]. Rio de Janeiro: SBA; 2020 [accessed on Apr 10, 2020]. Available at: https://www.sbahq.org/ebook/

12. Conselho Federal de Enfermagem (COFEN). Recomendações gerais para organização dos serviços de saúde e preparo das equipes de enfermagem: versão 2 [Internet]. Brasília: COFEN; 2020 [accessed on Apr 23, 2020]. 31 p. Available at: http://www.cofen.gov.br/wp-content/ uploads/2020/04/cofen_covid-19_cartilha_v3-4.pdf

13. Brasil. Ministério da Saúde. Secretaria de Ciência, Tecnologia, Inovação e Insumos Estratégicos em Saúde. Departamento de Gestão e Incorporação de Tecnologias e Inovação em Saúde. Diretrizes para diagnóstico e tratamento da COVID-19 [Internet]. Brasília: Ministério da Saúde; 2020 [accessed on Apr 10, 2020]. 74 p. Available at: https://portalarquivos. saude.gov.br/images/pdf/2020/April/13/Diretrizes-COVID-13-4.pdf

14. Correia, MITD, Ramos RF, Bahten LCV. Os cirurgiões e a pandemia do COVID-19. Rev Col Bras Cir [Internet]. 2020 [accessed on Apr 15, 2020];47:e20202536. Available at: http://www.scielo.br/scielo. php?script=sci_arttext\&pid=S0100-69912020000100601\&lng=en. https://doi.org/10.1590/0100-6991e-20202536
15. American College of Surgeons, American Society of Anesthesiologists, Association of periOperative Registered Nurses, American Hospital Association. Joint Statement: Roadmap for Resuming Elective Surgery after COVID-19 Pandemic [lnternet]. 2020 [accessed on Apr 20, 2020]. Available at: https://www.facs.org/covid-19/clinical-guidance/roadmap-elective-surgery

16. Kamer E, Çolak T. What to do when a patient infected with COVID-19 needs an operation: a Pre-surgery, Peri-surgery and Post-surgery Guide. Turk J Colorectal Dis [Internet]. 2020 [accessed on Apr 20, 2020];30:1-8. Available at: http://cms.galenos.com.tr/Uploads/Article_36547/turkishjcrd30-1-En.pdf. https://doi.org/10.4274/tjcd.galenos.2020.2020-3-7

17. Morrell ALG, Tustumi F, Morrell-Junior AC, Morrell AG, Ribeiro DMFR, Corsi PR, et al. Manejo intraoperatório em cirurgia laparoscópica ou robótica para minimizar a dispersão de aerossóis: Adaptações ao contexto da pandemia por COVID-19. Rev Col Bras Cir [Internet]. 2020 [accessed on Apr 20, 2020];47:e20202558. Available at: http:// www.revistadocbc.org.br/exportar-pdf/600/RCBC-2020-2558-en. pdf. https://doi.org/10.1590/0100-6991e-20202558

18. Zheng MH, Boni L, Fingerhut A. Minimally invasive surgery and the novel coronavirus outbreak: lessons learned in China and Italy. Ann Surg [Internet]. 2020 [accessed on Apr 20, 2020];272(1):e5e6. Available at: https://www.ncbi.nlm.nih.gov/pubmed/32221118. https://doi.org/10.1097/sla.0000000000003924

19. Tanner J, Parkinson H. Double gloving to reduce surgical crossinfection. Cochrane Database Syst Rev [Internet]. 2006 [accessed on Apr 15, 2020];2006(3):CD003087. Available at: https://www.ncbi. nlm.nih.gov/pubmed/16855997. https://doi.org/10.1002/14651858. cd003087.pub2

20. Association of periOperative Registered Nurses (AORN). Guidelines for Perioperative Practice: Transmission-Based Precautions [Internet]. Denver: AORN; 2018 [accessed on Apr 23, 2020]. Available at: https://aornguidelines.org/guidelines/ content?sectionid=173727681\&view=book\#205364548

21. Centers for Disease Control and Prevention (CDC). Interim U.S. guidance for risk assessment and public health management of healthcare personnel with potential exposure in a healthcare setting to patients with coronavirus disease (COVID-19) [Internet]. CDC; 2020 [accessed on Apr 20, 2020]. Available at: https://www.cdc.gov/ coronavirus/2019-ncov/hcp/guidance-risk-assesment-hcp.html

22. Association of periOperative Registered Nurses (AORN). Guidelines for Perioperative Practice: Environmental Cleaning (NEW) [Internet]. Denver: AORN; 2020 [accessed on Apr $15,2020]$. Available at: https://aornguidelines.org/guidelines/ content?sectionid $=173715702 \&$ view=book

23. Centers for Disease Control and Prevention (CDC). Information for Healthcare Professionals about Coronavirus (COVID-19) [Internet]. Atlanta: CDC; 2020 [accessed on Apr 24, 2020]. Available at: https:// www.cdc.gov/coronavirus/2019-ncov/hcp/caring-for-patients.html

24. Brasil. Resolução da Diretoria Colegiada - RDC n²22, de 28 de março de 2018 (BR). Regulamenta as Boas Práticas de Gerenciamento dos Resíduos de Serviços de Saúde e dá outras providências [Internet]. Brasil: Agência Nacional de Vigilância Sanitária; 2018 [accessed on Apr 20, 2020]. Available at: http:// portal.anvisa.gov.br/documents/10181/3427425/RDC_222_2018_. $\mathrm{pdf} / \mathrm{c5d} 3081 \mathrm{~d}-\mathrm{b} 331-4626-8448-\mathrm{c} 9 \mathrm{aa} 426 \mathrm{ec} 410$ 